\title{
On a Formula for the Jumps in the Semi-Fredholm Domain
}

\author{
VLADIMIR RAKOČEVIĆ
}

\begin{abstract}
In this paper we prove some properties of the lower s-numbers and derive asymptotic formulae for the jumps in the semi-Fredholm domain of a bounded linear operator on a Banach space.
\end{abstract}

\section{INTRODUCTION AND PRELIMINARIES}

In this note $X, Y, Z$ and $W$ are complex Banach spaces, and $B(X, Y)$ $(B(X))$ the set of all bounded linear operators from $X$ into $Y$ (on $X$ ). Let $K(X, Y)$ denote the set of compact linear operators from $X$ into $Y$ Let $U$ denote the closed unit ball of $X$. Let $T \in B(X, Y)$ and

$$
m(T)=\inf \{\|T x\|:\|x\|=1\}
$$

be the minimum modulus of $T$, and let

$$
q(T)=\sup \{\varepsilon \geq 0 \quad: \quad T U \supset \varepsilon U\}
$$

be the surjection modulus of $T$. Recall that both $m(T)$ and $q(T)$ are positive if and only if $T$ is invertible, and in this case $m(T)=q(T)=\left\|T^{-1}\right\|^{-1}$.

For each $r=1,2, \ldots, \infty$ we define the following lower analogues of the approximation numbers [8]:

$$
\begin{gathered}
m_{r}(T)=\sup \{m(T+F): \operatorname{rank} F<r\}, \\
q_{r}(T)=\sup \{q(T+F): \operatorname{rank} F<r\}, \\
g_{r}(T)=\max \left\{m_{r}(T), q_{r}(T)\right\} .
\end{gathered}
$$

1991 Mathematics Subject Classification: 47 A53, 47 A55.

Editorial Complutense. Madrid, 1992. 
If $M$ is a subspace of $X$, then $T_{\mid M}$ will denote the restriction of $T$ to $M . T$ is a semi-Fredholm operator if either the null space $N(T)$ is finite-dimensional and the range $R(T)$ is closed, or the codimension of $R(T)$ is finite. For such operators the index defined by

$$
\text { ind }(T)=\operatorname{dim} N(T)-\operatorname{codim} R(T) \text {, }
$$

and the minimum index by

$$
\min . \text { ind }(T)=\min \{\operatorname{dim} N(T), \operatorname{codim} R(T)\} \text {, }
$$

which is always finite. It was shown in [12, Theorem 8.3] that

$$
s(T)=\lim _{k} g_{\infty}\left(T^{k}\right)^{1 / k}
$$

is the semi-Fredholm radius of $T$, i.e. the supremum of all $\varepsilon \geq 0$ such that $T-\lambda I$ is semi-Fredholm for $|\lambda|<\varepsilon$. It is well known that the function min. ind $(T-\lambda I)$ is constant everywhere in the disk $|\lambda|<s(T)$ except possibly for a discrete subset $G$. We denote by $n(T)$ this constant, and call it the stability index of the semi-Fredholm operator $T[8]$. A point $\omega$ in $G$ is called a jumping point of the minimum index in the semi-Fredholm domain. For $\omega$ in $G$ we have min. ind $(T-\omega I)>n(T)$, and $X$ decomposes into the direct sum of two closed ' $T$-invariant subspaces $Y_{\omega}$ and $Z_{\omega}$, where $Z_{\omega}$ is finite-dimensional and $T-\omega I$ is nilpotent on it, while the restriction on $T-\lambda I$ to $Y_{\omega}$ has constant minimum index on a neighbourhood of $\omega$ [3, Theorem 4]. Consistently with the matrix case we define the (algebraic) multiplicity of the jumping point $\omega$ to be $\operatorname{dim} Z_{\omega}[8$, pp. 232]. Thus the point in $G$ can be ordered in such a way that

$$
\left|\omega_{1}(T)\right| \leq\left|\omega_{2}(T)\right| \leq \ldots<s(T)
$$

where each jump appears consecutively according to its multiplicity. If there are only $p(=0,1,2, \ldots)$ such jumps, we put $\left|\omega_{p+1}(T)\right|=\left|\omega_{p+2}(T)\right|=\ldots=s(T)$. Recall that [8, Theorem 1,1] if $T$ is a semi-Fredholm operator, then for each $r=1,2, \ldots$ we have

$$
\left|\omega_{r}(T)\right|=\lim _{k} g_{k n+r}\left(T^{k}\right)^{1 / k}
$$

where $n=n(T)$ is the stability index of $T$.

In this note we prove (1) when the stability index of $T$ is zero, and we believe that in this case the proof is simpler than the mentioned one in the general case. Further, we use a restriction techniques and show how this particular case is related to general case. 


\section{RESULTS}

In the following lemma we list some properties of the lower s-numbers.

Lemma 2.1. Let $T \in B(X, Y)$. Then

(i) $0 \leq m_{1}(T) \leq m_{2}(T) \ldots \leq m_{\infty}(T) \leq \sup _{\kappa \in K(X, Y)} \quad m(T+K) \leq \inf _{\kappa \in K(X, Y)}$ $\|T+K\|$,

(ii) $m_{n}(S+T) \leq m_{n}(S)+\|T\|$ for $S, T \in B(X, Y)$,

(iii) $m_{n}(R S T) \geq m(R) m_{n}(S) m(T)$ for $T \in B(X, Y), S \in B(Y, Z)$ and $R \in B(Z, W)$,

(iv) If $\operatorname{dim} X \geq n$, then $m_{n}(I)=1$,

(v) $m_{n+m-1}(S T) \geq m_{n}(S) m_{m}(T)$ for $T \in B(X, Y)$ and $S \in B(Y, Z)$,

(vi) $m_{n}(T)>0 \Leftrightarrow \operatorname{dim} N(T)<n, R(T)$ is closed and ind $(T) \leq 0$.

Proof. (i) By the definition and [6. pp. 389].

(ii) Let $F \in B(X, Y)$ and rank $F<n$. By [1, Lemma 2.2] we have

$$
m(S+T+F) \leq m(T+F)+\|S\| \leq m_{n}(T)+\|S\|,
$$

and hence $m_{n}(S+T) \leq m_{n}(T)+\|S\|$

(iii) Let $F \in B(Y, Z)$ and rank $F<n$. Now, $R F T \in B(X, W)$, rank $R F T<n$ and by $[1$, pp. 21$]$ we have

$$
m_{n}(R S T) \geq m(R(S+F) T) \geq m(R) m(S+F) m(T) .
$$

Further, it follows that $m_{n}(R S T) \geq m(R) m_{n}(S) m(T)$.

(iv) It is clear that $m_{n}(I) \geq 1$. If $m_{n}(I)>1$, then there is an $F \in B(X)$ and rank $F<n$, such that $m(I+F)>1$. Since $m(F)=0$, it follows that $m(I+F) \leq m(F)+\|I\|=1$, which is a contradiction. Hence $m_{n}(I)=1$.

(v) Let $F_{1} \in B(X, Y)$, rank $F_{1}<n, F_{2} \in B(Y, Z)$ and rank $F_{2}<m$. Then $\left(S+F_{2}\right)\left(T+F_{\nu}\right) \in B(X, Z),\left(S+F_{2}\right)\left(T+F_{\nu}\right)=S T+S F_{1}+F_{2}\left(T+F_{1}\right) \in B(X, Z)$ and rank $\left[S F_{1}+F_{2}\left(T+F_{1}\right)\right]<n+m-1$. Thus $m_{n+m-1}(S T) \geq m\left[\left(S+F_{2}\right)\right.$ $\left.\left(T+F_{1}\right)\right]>m\left(S+F_{2}\right) m\left(T+F_{1}\right)$, which proves $(v)$. 
(vi) Suppose that $m_{n}(T)>0$, rank $F<n$ and $\operatorname{dim} N(T) \geq n$. Now codim $N(F)<n$, and it follows that $N(T) \cap N(F) \neq\{0\}$. Thus $m(T+F)=0$, i.e., $m_{n}(T)=0$, whence a contradiction. Thus $m_{n}(T)>0$ implies dim $N(T)<n$. That $R(T)$ is closed and ind $(T) \leq 0$ follows by elementary properties of semi-Fredholm operators [9]. Conversely, if $R(T)$ is closed, dim $N(T)<n$ and ind $(T) \leq 0$, then by $[11$, Theorem $3.9(2)]$ there is an operator $F \in B(X)$ such that rank $(F)<n$ and $m(T+F)>0$. This implies that $m_{n}(T)>0$.

This completes the proof of the lemma.

Theorem 2.2. Let $T \in B(X)$ be a semi-Fredholm operator with the stabilitty index of $T$ equal to zero and $\min$. ind $(T-\lambda I)=\operatorname{dim} N(T-\lambda I)$ in the disk $|\lambda|<s(T)$ except possibly for the jumps $\omega_{r}(T), r=1,2, \ldots$. Then for each $r=1,2, \ldots$ we have

$$
\left|\omega_{r}(T)\right|=\lim _{k} m_{r}\left(T^{k}\right)^{l / k}
$$

Proof. We have to prove two things. First

$$
\left|\omega_{r}(T)\right| \leq \lim _{k} \text { inf } m_{r}\left(T^{k}\right)^{1 / k}
$$

and second

$$
\lim _{k} \sup m_{r}\left(T^{k}\right)^{1 / k} \leq\left|\omega_{r}(T)\right|
$$

Note that $\omega_{1}(T)=\lim m_{1}\left(T^{k}\right)^{1 / k}[4$, Theorem 3], and it is clear that (2) and (3) are true for $r=1$. To show the induction step for (2), take the least $q$ such that $\omega_{n-q}(T) \neq \omega_{n}(T)$. (If such a $q$ does not exist, then (2) is obvious since $\left|\omega_{n}(T)\right|=\left|\omega_{1}(T)\right|$ in that case). Let $Z$ be the direct sum of the finite-dimensional parts in the Kato decompositions corresponding to the points $\omega_{1}(T), \ldots$, $\omega_{n-q}(T)[3$, Theorem 4]. Now $\operatorname{dim} Z=n-q$. Let $Y$ be the intersection of the corresponding Kato complements to the finite-dimensional parts in the Kato decompositions corresponding to the points $\omega_{1}(T), \ldots, \omega_{n-q}(T)$. Thus the space $X$ decomposes into a direct sum of two closed subspace $Y$ and $Z$. These subspaces are $T$-invariant. Let $F$ be the removing operator from the proof of [12, Theorem 7.1], i.e., $F$ is zero on $Y$ and $\mu_{1} I$ on $Z ; \mu_{1}$ is any complex number with $\left|\mu_{1}\right|>\|T\|+s(T)$. By the proof of [12, Theorem 7.1] and [4, Theorem 3] we have that

$$
\lim _{k} m\left((T+F)^{k}\right)^{1 / k}=\left|\omega_{n-q+1}(T)\right|
$$


Further for each $k=1,2, \ldots$ we have

$$
m_{n}\left(T^{k}\right) \geq m_{n-q+1}\left(T^{k}\right) \geq m\left((T+F)^{k}\right),
$$

and so the proof of (2) is complete.

Now we turn to prove the inequality (3). Let $W$ be the direct sum of the finite-dimensional parts in the Kato decompositions corresponding to the points $\omega_{1}(T), \ldots, \omega_{n}(T)[3$, Theorem 4]. Now $\operatorname{dim} W \geq n$. Let $V$ be the intersetion of the corresponding Kato complements to the finite-dimensional parts in the Kato decompositions corresponding to the points $\omega_{1}(T), \ldots, \omega_{n}(T)$. Thus the space $X$ decomposes into a direct sum of two closed subspaces $W$ and $V$. These subspaces are $T$-invariant. Let $F \in B(X)$ and rank $F<n$. Hence, there is a vector $h \in W \cap N(F)$ such that $h \neq 0$. Let $P$ be the projection of $X$ onto $W$ along $V$. Then

$$
\|(T+F) h\|=\|T h\|=\|T P h\| \leq\left\|T_{\mid W \|}\right\| P\|\| h \| .
$$

Thus, $m(T+F) \leq\|P\|\left\|T_{\mid w \|}\right\|$. It is easy to see that for each $k=1,2, \ldots$ we have $m\left(T^{k}+F\right) \leq\|P\|\left\|T^{k}{ }_{1}\right\|$. Consequently $m_{n}\left(T^{k}\right) \leq\|P\|\left\|T^{k}{ }_{\mid w}\right\|$, and since the spectral radius of $T_{\mid W}$ is equal to $\left|\omega_{n}(T)\right|$, it follows that

$$
\lim _{k} \sup m_{n}\left(T^{k}\right)^{1 / k} \leq\left|\omega_{n}(T)\right|
$$

This proves (3), and the proof of the theorem is complete.

Remark 2.3. Let us mention that if in Theorem 2.2 we have that $\omega_{1}(T) \neq 0$, then we can prove (3) in the following way (we use the same notations as in the proof of Theorem 2.2): Now $T_{1 W}: W \rightarrow W$ is invertible and since $\operatorname{dim} W \geq n$ we have by Lemma 2.1 (iv) that $m_{n}\left(T^{k}\left(T_{\mid W^{-k}}\right)\right)=1, k=1$, $2, \ldots$. Thus by Lemma $2.1(v)$ we have $\left.1 \geq m_{n}\left(T^{k}\right) m\left(T_{\mid W^{-1}}\right)^{k}\right)$, and so

$$
\left.m_{n}\left(T^{k}\right) \leq 1 / m\left(T_{\mid W^{-1}}\right)^{k}\right)=\| T_{\left|W^{k}\right|} .
$$

Since the spectral radius of $T_{\mid W}$ is equal to $\left|\omega_{n}(T)\right|$ we conclude that

$$
\lim _{k} \sup m_{n}\left(T^{k}\right)^{1 / k} \leq\left|\omega_{n}(T)\right|
$$

whence the result.

Next we state properties of $q_{n}(T)$ and the dual result of Theorem 2.2. They can be proved similarly, so we leave out details. 
Lemma 2.4. Let $T \in B(X, Y)$. Then
(i) $0 \leq q_{1}(T) \leq q_{2}(T) \ldots \leq q_{\infty}(T) \leq$ $\|T+K\|$.
$\sup _{K \in K(X, Y)} q(T+K) \leq \inf _{K \in K(X, Y)}$
(ii) $q_{n}(S+T) \leq q_{n}(S)+\|T\|$ for $S, T \in B(X, Y)$,
(iii) $q_{n}(R S T) \geq q(R) q_{n}(S) q(T)$ for $T \in B(X, Y), \quad S \in B(Y, Z)$ and $R \in B(Z, W)$,
(iv) If $\operatorname{dim} X \geq n$, then $q_{n}(I)=1$,
(v) $q_{n+m-1}(S T) \geq q_{n}(S) q_{m}(T)$ for $T \in B(X, Y)$ and $S \in B(Y, Z)$,
(vi) $q_{n}(T)>0 \Longleftrightarrow \operatorname{codim} R(T)<n$, and ind $(T) \geq 0$,
(vii) If $m_{n}(T)>0$ and $q_{n}(T)>0$, then $m_{n}(T)=q_{n}(T)$ and ind $(T)=0$.

Proof. We shall prove only (vii). From (vi) and Lemma 2.1 (vi), it follows that $\operatorname{dim} N(T)<n, R(T)$ is closed, codim $R(T)<n$ and ind $(T)=0$. Let $F \in B(X, Y)$ and rank $F<n$. If $m(T+F)>0$, then $\operatorname{dim} N(T+F)=0$, and it follows that codim $R(T+F)=0$. Thus, $m(T+F)=q(T+F) \leq q_{n}(T)$, and we have that $m_{n}(T) \leq q_{n}(T)$. In a similar way, we can prove that $q_{n}(T) \leq m_{n}(T)$, and the proof is complete.

Theorem 2.5. Let $T \in B(X)$ be a semi-Fredholm operator with the stability index of T equal to zero and min. ind $(T-\lambda I)=\operatorname{codim} R(T-\lambda I)$ in the disk $|\lambda|<s(T)$ except possibly for the jumps $\omega_{r}(T), r=1,2, \ldots$. Then for each $r=1,2, \ldots$ we have

$$
\left|\omega_{r}(T)\right|=\lim _{k} q_{r}\left(T^{k}\right)^{1 / k} .
$$

Proof. By Lemma 2.4 and Theorem 2.2.

For $T^{\text {in }} B(X)$ set $N\left(T^{\infty}\right)=\cup N\left(T^{m}\right)$ and $R\left(T^{\infty}\right)=\cap R\left(T^{n}\right)$. If $T$ is a semiFredholm, then it is well known ([5, Theorem 4.1] see also [7, Theorem 5.2] for general case) that the function $\lambda \rightarrow N\left((T-\lambda)^{\infty}\right)+R\left((T-\lambda)^{\infty}\right)$ is constant, say $W$ everywhere in the disk $|\lambda|<s(T)$. Let us remark that $W$ is closed, hence Banach subspace in $X$ (see ([5, pp. 517, Corollary 3.2$]$ and [10, Proposition 1.10]) or ([7, Remark 5.3] and [2, Lemma 3.6 (a), Theorem 3.8])) The restriction of $T$ to the subspace $W$ has been studied in [2], [5], [7] and [10]. Now we have 
Theorem 2.6. Let $T \in B(X)$ be a semi-Fredholm operator, and $\omega_{r}(T)$, $r=1,2, \ldots$ are as above. Then for each $\omega_{r}(T), r=1,2, \ldots$ we have

$$
\left|\omega_{r}(T)\right|=\lim _{k} q_{r}\left(\left(T_{\mid W}\right)^{k}\right)^{1 / k}
$$

Proof. By [5, Theorem 4.1] and [3, Theorem 4] we know that everywhere in the disk $|\lambda|<s(T)$ we have that $W=R\left((T-\lambda)^{\infty}\right) \oplus N_{\lambda}$, where $N_{\lambda}$ is finite dimensional subspace $T$-invariant and $(T-\lambda)_{\mid N_{\lambda}}$ is nilpotent on it (see also [7, Remark 5.3]). Thus by [2, Theorem 3.4] we have that $(T-\lambda)$ $(W)=(T-\lambda)\left(R\left((T-\lambda)^{\infty}\right) \oplus N_{\lambda}\right)=R\left((T-\lambda)^{\infty}\right) \oplus(T-\lambda)\left(N_{\lambda}\right)$. Thus, $(T-\lambda)_{1 W}$ is semi-Fredholm, $\operatorname{dim} W / R\left((T-\lambda)_{\mid W}\right)<\infty$ and the stability index of $T_{\mid W}$ is zero ([5, Proposition 2.6]). Let us remark that $\omega_{r}(T), r=1,2, \ldots$ are jumps (with the same multiplicity) in the semi-Fredholm region of $T_{\mid w}$. Now the proof of the theorem follows by Theorem 2.5 .

Acknowledgements. I am grateful to Professor Jaroslav Zemánek for helpful conversations. The author also thanks the referee for helpful comments and suggestions concerning the paper.

\section{References}

[1] H. A. GINDLER and A. E. TAYLOR: The minimum modulus of a linear operator and its use in spectral theory. Studia Math. 22(1962), 15-41.

[2] S. GRABINER: Uniform ascent and descent of bounded operators, J. Math. Soc. Japan, 34 (1982), 317-337.

[3] T. KATO: Perturbation theory for nullity, deficiency and other quantities of linear operators, J. Analyse Math., 6(1958), 261-322.

[4] JR., E. MAKAI and J. ZEMÁNEK: The surjectivity radius, packing numbers and boundedness below of linear operators. Integral Equations and Operator Theory, 6(1983), 372-384.

[5] M. O. SEARCOID and T. T. WEST: Continuity of the generalized kernel and range of semi-Fredholm operators, Math. Proc. Cambridge Philos. Soc. 105 (1989), 513-522.

[6] V. RAKö̌́VIć: On one subset of $M$. Schechter's esential spectrum, Mat. Vesnik, 33 (1981), 389-391.

[7] V. RAKOČEvić: Generalized spectrum and commuting compact perturbations, Proc. Edinburgh Math. Soc. (to appear).

[8] V. RAKOČEVIĆ and J. ZEMÁNEK: Lower s-numbers and their asymptotic behaviour, Studia Math. 91 (1988), 231-239.

[9] M. SCHECHTER: Principles of functional analysis, Academic Press, Student edition, New York and London, 1973.

[10] T. T. West: $\dot{A}$ Riesz-Schauder theorem for semi-Fredholm operalors, Proc. Roy. Irish Acad. Sect. A 87 (1987), 137-146. 
[11] B. YooD: Properties of linear transformations preserved under addition of completely continuous transformation, Duke Math. J. 18 (1951), 599-612.

[12] J. ZEMANEK: Geometric characteristics of semi-Fredholm operators and their asymptotic behaviour, Studia Math. 80(1984), 219-234.

University of Niš, Faculty of Philosophy

Department of Mathematics

Cirila and Metodija 2

Recibido: 27 de septiembre de 1991

Yugoslavia

Revisado: 18 de enero de 1992 